\title{
AN INVITATION TO THE MEDICAL STUDENTS OF THE WORLD TO JOIN THE GLOBAL COALITION TO IMPROVE CARE FOR CHILDREN AND ADULTS WITH CONGENITAL HEART DISEASE ACROSS THE WORLD
}

\author{
Pierre-Luc Bernier ${ }^{1}$, Noritaka Ota ${ }^{2}$, Christo I. Tchervenkov ${ }^{1 *}$, Jeffrey P. \\ Jacobs $^{3}$, Giovanni Stellin 4 , Hiromi Kurosawa ${ }^{5}$, Constantine Mavroudis ${ }^{6}$, \\ Sertac Cicek ${ }^{7}$, Zohair Al-Halees ${ }^{8}$, Martin Elliott ${ }^{9}$, Marcelo Jatene ${ }^{10}$, Richard \\ A. Jonas ${ }^{11}$, Rob Kinsley ${ }^{12}$, Christian Kreutzer13 ${ }^{3}$, Juan Leon-Wyss ${ }^{14}$, Jinfen \\ Liu $^{15}$, Bohdan Maruszewski ${ }^{16}$, Graham Nunn ${ }^{17}$, Samuel Ramirez-Marroquin ${ }^{18}$, \\ Nestor Sandoval ${ }^{19}$, Shunji Sano ${ }^{20}$, George Sarris ${ }^{21}$, Rajesh Sharma ${ }^{22}$, Thomas \\ Spray $^{23}$, Ross Ungerleider ${ }^{24}$, Hervé Yangni-Angate ${ }^{25}$, Gerhard Ziemer ${ }^{26}$
}

\section{INTRODUCTION}

Medical schools teach their students about anatomy, histology, cell biology, genetics, embryology, physiology, biochemistry, pathophysiology, pharmacology, and other subdivisions of the medical knowledge and know-how. Despite acquiring this complex science and having the opportunity to interact with patients in the clinical setting, very rarely do medical students gather a global understanding of what impact a disease represents on a personal, societal and global level. Congenital heart disease (CHD) is a condition that is present in approximately 1 in 100 births, has a remarkable heterogeneity and complexity and has a significant impact on the child's survival and quality of life if left untreated. Even when the initial congenital defect is successfully repaired, patients with CHD require a lifelong follow-up and the possibility of subsequent reinterventions. Physicians are involved from the beginning to the end with the diagnosis, counselling, every step of the treatment and the lifelong follow-up. The treatment of CHD requires an enormous

*To whom correspondence should be addressed:

Christo I. Tchervenkov, M.D.

The Montreal Children's Hospital

Cardiovascular Surgery,

2300, Tupper street, Room C-829

Montreal, Quebec, Canada

H3H 1P3

Email: ctchervenkov@wspchs.org

Website: www.wspchs.org effort, resources and the participation of a huge team made up of a multitude of highly trained medical specialists, such as cardiac surgeons, cardiologists, cardiac anaesthetists, cardiac intensivists and many others, who often have dedicated their entire professional lives to this exciting field of medicine.

Far from claiming to be a thorough and exhaustive exposé on $\mathrm{CHD}$, this article rather aims to present to medical students from everywhere in the world an overview of the nature of this important disease and of its global impact. We will provide a definition of CHD, highlight a few examples, give a summary of its epidemiology, and finally summarize the history of the study and treatment modalities. Our focus will also be on the inequalities in the treatment of CHD between the western world and developing nations. Finally, the challenges that must be addressed to truly improve care around the world will be presented.

\section{DEFINITION OF CONGENITAL HEART DISEASE}

What is congenital heart disease? Simply put, one could say that it is a developmental malformation of the heart started during fetal development and present at birth, which results in abnormal anatomy and physiology of the heart, has a profound deleterious impact on the other organs and structures of the body and adversely affects the health of the child. Embryologically, it results from a multitude of factors, 
most of them unknown, which lead to abnormalities in the in-utero development of the fetal heart. Anatomically, CHD comprises a huge number of cardiac malformations from the simplest to the most complex, characterized by extreme heterogeneity, which alter the normal and optimal structure and function of the heart and of other organs in the body.

Every part of the heart and great vessels can be involved and malformed. The atria, ventricles, the great vessels, the atrioventricular and semilunar valves, and the coronary arteries can be affected to various degrees depending on the specific heart malformation present.

The many deleterious consequences affecting other organs further complicate CHD. As a whole, CHD can be present in many forms and across the entire age spectrum; from the blue newborn baby with profound cyanosis due to transposition of the great arteries to the old adult with progressive fatigue due to a previously undiagnosed atrial septal defect coming to medical attention with overt heart failure. Although considered by many a rare disease, CHD is the most common congenital illness and presents a significant burden to the children of this world and to mankind.

\section{CHILDREN OF THE WORLD AFFECTED BY CONGENITAL HEART DISEASE}

Globally, approximately 130 million babies are born every year. It is said that CHD affects approximately $0.8 \%$ of all live births. Simple calculations indicate that approximately 1 million babies are born each year with CHD and $90 \%$ of those newborns see the world in areas of the globe where access to appropriate medical care is either difficult or impossible. One can estimate that of the 280000 babies who die each year from a congenital cardiac anomaly in the neonatal period, more than 250 000 are not offered the care that has been available over the past half-century and that has allowed cardiac surgeons and cardiologists from around the world to save countless lives, very often with fairly simple measures [1].

Listing every congenital lesion affecting the human heart is far beyond the scope of this paper. However, it is interesting to briefly ponder upon the natural history of some of those malformations to understand adequately their significance. The progression and severity of the disease is specific to every lesion and present in a wide spectrum. For example, individuals with large atrial septal defects face a risk of mortality of $5-15 \%$ before the age of 30 years. Similarly, the presence of a patent ductus arteriosus is associated with a $30 \%$ risk of mortality before the age of one year and a $42 \%$ risk of death before 45 years of age. Of all the individuals born with tetralogy of Fallot with pulmonary stenosis, $25 \%$ will die within their first year of life if left untreated. $40 \%, 70 \%$, and $95 \%$ will be dead, respectively, by the age of 3,10 , and 40 years. Looking at more complex pathologies, transposition of the great arteries is associated, all varieties considered, with a $45 \%, 85 \%$, and $90 \%$ mortality at one month, 6 months and one year respectively. Finally, hypoplastic left heart syndrome, which is the fourth most common congenital cardiac defect, results in the mortality of most neonates within one to two weeks of birth and survival beyond 6 weeks of age is unusual [2].

CHD is a major burden to the children of this world. However, despite being rather revealing, the mortality rates stated above do not give an accurate rendering of the misery children and young adults with CHD face. Indeed, the morbidity ensuing from unrepaired CHD is difficult to truly gauge and quantify but is immensely important. Therefore, one must consider with equal magnitude the decreased quality of life faced by those individuals. Suffering from frequent pulmonary infections, a high risk of bacterial endocarditis, chronic cyanosis, the risk of neurological events and decreased functional status, the existence of a child surviving with unrepaired CHD is far from normal and optimal.

\section{HISTORY OF DIAGNOSIS AND TREATMENT OF CONGENITAL HEART DISEASE}

In 1936, Dr. Maude Abbott (1869-1940), of McGill University in Montreal, published her famous Atlas of congenital cardiac disease. This work, proposing the first classification system for CHD, was based on her lifelong study of the clinical and post-mortem records of more than 1000 cases of cardiac malformations. The Atlas rapidly became the main international reference in CHD and Maude Abbott has since been considered the founder of the systematic study of CHD [3]. The development of the study of congenital cardiac disease quickly flourished thereafter. Maverick surgeons now remembered for their great sense of innovation paved the way to the successful treatment of CHD. An important milestone in the treatment of CHD was the development of a palliative operation to alleviate the profound cyanosis in patients with tetralogy of Fallot. This was the result of a historic collaboration between Alfred Blalock, Helen Taussig and Vivien Thomas, with the advent of the systemic pulmonary shunt bearing their name. However, the single most important step in the treatment of CHD was the invention of the heartlung machine by John Gibbon Jr of Jefferson Medical College in Philadelphia who spent 20 year working on its development and was the first to use it successfully in a human in 1953. Subsequently, with the pioneering efforts of physicians and surgeons it soon became 
possible to repair even complex cardiac malformations and consequently impact on the life of millions of children. Steady progress in diagnostic modalities, in surgical techniques, in establishment of new procedures, as well as improvement in preoperative care, intra-operative anaesthesia and perfusion management and intensive post-operative care have resulted in significant reduction in mortality and morbidity. Major improvement in early and late survival for CHD also ensued. Complex malformations such as transposition of the great arteries, tetralogy of Fallot, and hypoplastic left heart syndrome can now be treated with real optimism for the future lives of the afflicted children. However, much work needs to be done with the rapidly increasing number of adults with $\mathrm{CHD}$ and the realization of the long-term problems in many of the "repaired" CHD patients.

\section{CURRENT STATUS IN TREATMENT FOR CONGENITAL HEART DISEASE}

While we have some information regarding the status of congenital cardiac care in North America and the rest of the western world, little is known about the precise situation in the remainder of the globe.

Indeed, data published in 2005 by Jacobs and al. in the Report of the 2005 Society of Thoracic Surgeons Congenital Heart Surgery Practice and Manpower Survey gives a good picture of the state of our specialty in North America. At the time of the survey, there were 248 congenital cardiac surgeons in the United States of America and 15 in Canada. Each group is respectively distributed amongst 121 and 8 surgical centres. The average age of a congenital cardiac surgeon in North America is 48.3 years and, on average, surgeons have 9.2 years of post-graduate training. Only $4 \%$ of all congenital cardiac surgeons in North America are females. Based on those numbers, there is approximately one congenital cardiac surgeon per 1.5-2 millions population [4].

This information is available concerning North America, but data is scarce regarding the rest of the world. To palliate to this situation, the World Society for Pediatric and Congenital Heart Surgery (www.wspchs.org) embarked on a fact-finding mission in 2007. Preliminary results from the survey conducted confirm an important inconsistency between the various regions of the world. While western countries enjoy a proportion of approximately 1 congenital cardiac surgeon per 1.5-2 million inhabitants, our survey shows very different ratios in South America, Asia, and Africa; respectively $1: 6$ million, $1: 25$ million, and $1: 38$ million. Quick calculations, which are obviously limited by their inaccuracy and the imprecise nature of the data from which they are based on, indicate that the developing world is in need of approximately 1000 to 1500 additional congenital cardiac surgeons. This is a gross estimate which is, nonetheless, very revealing.

The data currently being gathered by the World Society is showing that the patterns of practice are otherwise quite similar on the five continents. Indeed, the average age, the proportions of males and females, and the duration of post-graduate training of congenital cardiac surgeons are quite comparable across the board. However, the funding sources of congenital cardiac surgery programs vary. In the United States, for example, most units are privately funded. However, in the rest of the world, it appears that government sources are the main financial supporters of congenital cardiac surgery centers. This information is incomplete and the World Society is planning to pursue its fact-finding effort in this regard.

The most important component of the treatment of CHD is teamwork. The concerted input of many specialists, i.e. surgeons, cardiologists, intensivists, anesthesiologists, perfusionists, nurses is needed. Although in a congenital cardiac surgery program the surgeon often assumes a leadership role, the collaboration of all is essential to its success. Indeed, the necessary diagnostic modalities, such as echocardiography, angiography, computerized tomography, or magnetic resonance imaging are often difficult to evaluate and require the analytical and judgment skills of specifically trained physicians. The same is true for the intra-operative management of the patients. Indeed, cardiac anesthesiologists and perfusionists with specialized training in the care of children with congenital cardiac disease carry the utmost importance. This concept carries over to the intensive care unit with the post-operative care of the patients. The treatment of CHD is very complex and necessitates the work of many highly skilled individuals, a high level of organization and logistics and appropriate financial support

\section{CHALLENGES TO IMPROVEMENT OF CARDIAC CARE FOR CONGENITAL HEART DISEASE ACROSS THE WORLD}

For an obvious reason of lack of resources, the development of pediatric and congenital heart surgery around the world has been late, slow and erratic in most developing countries. Competing priorities, poor structural organizations, lack of financial resources, lack of trained personnel and absence of stable training and educational infrastructure are among the many reasons for this unfortunate situation. While some countries have succeeded in developing surgical programs to treat $\mathrm{CHD}$, most children born with a cardiac malformation still do not have access to 
appropriate medical and surgical care.

To palliate to this complete or relative absence of access to cardiac surgical care in many countries, different projects have emerged. This help takes many forms and it is safe to say that the perfect model does not exist yet. Most of this work is funded by charitable organizations and helps children with CHD on a small scale. Some groups bring children in need to North America or Europe so that they can undergo surgery and then return to their country of origin. This has been largely inefficient due to the great cost and effort required and has only helped very few children. Humanitarian surgical missions consisting of cardiac surgeons, cardiologists, intensivists, anesthesiologists, perfusionists, and nurses visit countries for a few weeks at a time with the goals to operate children in need of surgery as well as to form surgical teams and hopefully launch congenital cardiac surgery programs which can flourish and progress in a sustainable fashion. Finally, some areas have seen the establishment of cardiac centres after the local training of their healthcare professionals or their return from instruction abroad. Surgeons, cardiologists, anaesthesiologists, intensivists and other professionals trained internationally infinitely enrich their countries upon their return. However, a serious problem is the lack of retention due to the poor local infrastructure and critical mass of health care professionals, which further exacerbates the vicious circle. By and large these efforts have so far had a minimal impact in the numbers of children treated compared to the overall needs.

All the efforts described above have to be concerted and the various groups interested in globally improving congenital cardiac care around the world have to meet and organize themselves to reach their goal in a sustainable fashion. The World Society for Pediatric and Congenital Heart Surgery aims to provide this forum. It is of paramount importance to identify the most profitable, practical, and cost-efficient way to help the children of the world with CHD.

Indeed, while we do intensive research and strive to decrease our mortality rates by fractions of percentage points in the western world, the vast majority of children born with CHD still have an unacceptably high risk of mortality. The progress of the last decades has been largely limited to the developed world. Consequently, every year approximately $90 \%$ of more than 1000000 children born with CHD across the world receive either suboptimal care or are totally denied care.

Although CHD has not been on the radar screen of most global health agencies and humanitarian organizations so far and since there has been little focus on the global epidemiology of pediatric and congenital cardiac disease, considerable work remains to be done if one is to try to significantly improve cardiac care around the globe.

\section{WORLD SOCIETY FOR PEDIATRIC AND CONGENITAL HEART SURGERY: LEADING THE WAY IN A NEW PARADIGM SHIFT FOR THE GLOBAL IMPROVEMENT OF CARE}

The idea for a global organization was first discussed in 2004 in Montreal during an international meeting of pediatric and congenital heart surgeons scheduled during the Centennial Celebrations of the Montreal Children's Hospital under the leadership of Christo I. Tchervenkov, Professor of Surgery at McGill University. The World Society for Pediatric and Congenital Heart Surgery was then established in 2006.

During his Presidential Address at the Inaugural Meeting of Washington DC in May 2007, the World Society President, Christo I. Tchervenkov from Montreal, Canada, presented his vision for the role of the World Society and talked about the key elements for the global improvement of care. President Tchervenkov coined the phrase "Medicine of RESPECT" or "Medicine of Responsible Education Sustained through Partnership, Empowerment, Care and Commitment, and Teamwork and Trust" as the basis of the necessary paradigm shift required for the global improvement of care in the 21 st century [5].

The World Society for Pediatric and Congenital Heart Surgery is in an exceptional position to truly improve care for pediatric and congenital cardiac disease across the world. Being the largest society for pediatric and congenital heart surgery in the world with more than 500 members from close to 70 countries, its potential for playing a defining role in the global improvement of care is significant. The vision of the World Society is that every child born anywhere in the world with a congenital heart defect should have access to appropriate medical and surgical care. Its mission is to promote the highest quality comprehensive cardiac care to all patients with pediatric and/or congenital heart disease, from the fetus to the adult, regardless of the patient's economic means, with an emphasis on excellence in education, research and community service. The vision and mission of the World Society are being pursued by working towards clearly defined objectives in the following domains: patient care, training and education, research, and community service. In the area of patient care, the World Society hopes to promote the professional and educational development of surgeons specializing and practicing pediatric and congenital heart surgery across the world, as well as the dissemination of informational support to patients, parents of patients, families of patients, and 
health care professionals, working in collaboration with societies such as the International Society for Nomenclature of Pediatric and Congenital Heart Disease. It also wants to develop global standards for the training and education of pediatric and congenital heart surgeons and for the practice of pediatric and congenital heart surgery. Finally, its members originating from all areas of the globe will form a forum for the respectful exchange of knowledge in the form of scientific meetings and publications across the world

Finally, and maybe most importantly, the World Society aims to foster collaboration across medical and surgical subspecialties and is working toward the establishment of a multi-societal Global Organization for Pediatric and Congenital Heart Disease. This Organization has seen birth in June 2008, in Montreal, with the proclamation of a resolution for its establishment at the World Summit on Pediatric and Congenital Heart Surgery Services, Education and Cardiac Care in Children and Adults with Congenital Heart Disease taking place in Montreal from June 1922, 2008. Composed of multiple professional associations of surgeons, cardiologists, intensivists, anaesthesiologists, nurses, and perfusionists, this Global Organization will be the cornerstone of the large-scale improvement of congenital cardiac care around the world.

\section{CONCLUSION}

It is our hope that this article will give a good overview of the nature of CHD, of its global impact and of the challenges that inevitably need to be addressed in order to truly impact on the future on innumerable children around the world. Every speciality concerned by CHD need to be empowered and embark on this journey to globally improve our services to children of countless developing countries.

Congenital cardiac surgery has evolved enormously over the last 60 years since the pioneering work of Abbott, Blalock, Taussig, Gibbons, Lillehei, Kirklin, Castaneda and others. It has matured to a point where it enjoys unprecedented successes with results barely conceivable only a few decades ago. However, only a small proportion of the children born yearly with CHD benefit from those successes. The world needs a major paradigm shift in the care for pediatric and congenital cardiac disease. The field of congenital cardiac surgery does not need any new major scientific discovery. There is rather a need to share with the rest of the world what has already been discovered and implemented over the last half-century. A hand needs to be extended to those in the developing world. Such major improvement in care can only result from the systematic organization of all health care providers across the world, based on the concept of a Medicine of RESPECT, or the Respectful Education Sustained through Partnership, Empowerment, Care, Commitment, Teamwork, and Trust [5].

Medical students, being where they are in their training, should hopefully be influenced and enlightened by the facts previously described. Their open-mindedness, optimistic view of the world, and indefectible enthusiasm represent perhaps what is needed to truly advance this agenda. The objectives that the World Society for Pediatric and Congenital Heart Surgery has given itself are numerous and broad and will likely have a real impact on the remaining $90 \%$ of children born with CHD who are not offered treatment. However, the process we have embarked on will be long and arduous and may be filled with many frustrations. It will not be a sprint, or even a marathon, but perhaps more of a long arduous triathlon. Nevertheless, we have taken the first few steps on this challenging and exciting journey. The World Society for Pediatric and Congenital Heart Surgery and the soon to be established Global Organization for Pediatric and Congenital Heart Disease are in a unique position to lead the way in this seemingly daunting task. We sincerely welcome the input of all medical students and strongly encourage all of those interested to participate, in one way or another, in their upcoming careers or academic projects, to the furthering of this somewhat grandiose, but, in our opinion, noble and realistic endeavour.

\section{REFERENCES}

1. Lawn JE, Cousens S, Zupan J; Lancet Neonatal Survival Steering Team. 4 million neonatal deaths: When? Where? Why? Lancet 2005; 365: 891-900.

2. Kirklin JW, Barratt-Boyes BG. Cardiac Surgery, 2nd edition. New York: Churchill-Livingstone, 1993.

3. Abbott ME. Atlas of Congenital Cardiac Disease, (re-edition). McGill-Queen's University Press 2006. 62 pages.

4. Jacobs ML, Mavroudis C, Jacobs JP, Tchervenkov CI, Pelletier GJ. Report of the 2005 STS Congenital Heart Surgery Practice and Manpower Survey. Ann Thorac Surg. 2006 Sep;82(3):11528 .

5. Tchervenkov CI. Presidential Address: Hearts for Life across the World. The World Society News 2007. Volume 1, Issue 1, pages 6-8. 
Pierre-Luc Bernier completed his medical studies at McGill University (Montreal, Canada) and is currently enrolled in the Cardiac Surgery training program at this institution. He held the position of Research Fellow for the World Society for Pediatric and Congenital Heart Surgery from 2007-2008. He has a strong interest in congenital heart surgery and is still very active within the World Society.

\section{AUTHOR AFFILIATIONS:}

1. Cardiac Surgery, Montreal Children's Hospital, Montreal, Québec, Canada.

2. Cardiac Surgery, Shizuoka Children's Hospital, Shizuoka, Japan

3. Division of Thoracic and Cardiovascular Surgery, The Congenital Heart Institute of Florida, All Children's Hospital / Children's Hospital of Tampa, University of South Florida College of Medicine, Cardiac Surgical Associates, Saint Petersburg and Tampa, Florida, United States of America

4. Pediatric Cardiac Surgery Unit, University of Padova Medical School, Padova, Italy.

5. Cardiovascular Surgery, Heart Institute of Japan, Tokyo Women's Medical University, Tokyo, Japan.

6. Department of Surgery, Division of Cardiovascular-Thoracic Surgery, Northwestern University Medical School, Children's Memorial Hospital, Chicago, Illinois, USA.

7. Anadolu Health Center ASM Hospital Heart Institute, Istanbul, Turkey.

8. King Faisal Heart Institute, King Faisal Specialist Hospital and Research Centre, Riyadh, Saudi Arabia.

9. Cardiothoracic Surgery, Hospital for Sick Children, Great Ormond Street, London, UK.

10.Department of Pediatric Cardiac Surgery and Pediatric Cardiology, Heart Institute, University of São Paulo Medical School, SP, São Paulo, Brazil.

11. Department of Cardiac Surgery, Children's National Heart Institute, Children's National Medical Center, Washington, DC, USA.

12. Department of Cardiac Surgery, Sunninghill Hospital, Johannesburg, South Africa.

13. Division of Cardiac Surgery, Hospital de Niños Ricardo Gutierrez, Buenos Aires, Argentina.

14. Department of Pediatric Cardiac Surgery of Guatemala, UNICAR, Guatemala City, Guatemala.

15. Department of Pediatric Thoracic and Cardiovascular Surgery, Xinhua Hospital, Shanghai Children's Medical Center, Shanghai Second Medical University, Shanghai, China.

16. Cardiac Surgery, Memorial Hospital Child's Health Centre, Warsaw, Poland.

17. The Children's Hospital at Westmead, Westmead, NSW, Sydney, Australia.

18. Department of Cardiac Surgery, Instituto Nacional de Cardiología Ignacio Chavez, Mexico City, Mexico.

19. Department of Cardiovascular Surgery, Fundacion Cardioinfantil, Bogotá, Colombia.

20. Department of Cardiovascular Surgery, Okayama University Hospital, Okayama, Japan.

21. Department of Pediatric and Congenital Heart Surgery, Mitera and Hygeia Hospitals, Athens, Greece.

22. Escorts Heart Institute and Research Centre, New Delhi, India.

23. The Children's Hospital of Philadelphia, University of Pennsylvania School of Medicine, Philadelphia, Pennsylvania, USA.

24. Doernbecher Children's Hospital, Oregon Health Sciences University, Portland, Oregon, USA.

25. Department of Thoracic and Cardiovascular Surgery, Bouake Teaching Hospital, Abidjan, Ivory-Coast.

26. Department of Thoracic, Cardiac and Vascular Surgery, University of Tubingen, Tubigen, Germany. 